\title{
DER ORT DES GESCHEHENS
}

\author{
„Die anderen Festungen Frankreichs schützen die Provinzen, \\ Metz aber schützt den Staat.“
}

Vauban $^{1}$

Metz ist das Herz Lothringens und seit ältester Zeit ein wichtiger Straßenkreuzungs- und Verkehrsknotenpunkt am Rande des gallo-römischen Kulturkreises. Der Moselfluß teilt Stadt und Region und liegt wie ein breiter Graben zwischen den umliegenden Höhen. Jahrhunderte war er die wichtigste Lebensader der Messins. Die sandige Hügellandschaft an seinen Ufern taugte vornehmlich zum Weinbau, und den Bauern und Winzern brachte vor allem der per Schiff betriebene Handel, den bereits die Römer eifrig gefördert hatten, Lohn und Brot. Man brauchte die offenen Wege ins Land, denn die Stadt war seit jeher „Lager und Depot “2, ein Sammel- und Umschlagplatz für Waren, Geld und Menschen. Immer strömten hier die Dinge zusammen, traf und begegnete man sich. Wo heute ein weitverzweigtes Autobahnkreuz liegt, gab es zur Belagerungszeit zwei Bahnlinien, eine südliche von Nancy und eine west-östliche von Paris nach Saarbrücken. Mit den Eisenbahnen, einer hier erst relativ spät eingeführten Neuerung ${ }^{3}$, kam die Industrialisierung, und ganz neue Mobilitätschancen, gerade im militärischen Bereich, taten sich auf. Jedoch sollte der Gütertransport per Schiene im Herbst 1870, wir werden darauf später näher eingehen, vor der Festung noch kaum wie gewünscht funktionieren. Für die Menschen in der Stadt blieb das neue Verkehrsmittel, wie überhaupt jede Verbindung mit dem Umland, während der Belagerungszeit ohnehin gänzlich gesperrt. Erst die gefangengenommene Rheinarmee machte Anfang November wieder Bekanntschaft mit jenen unbequemen, kalten Zügen, die sie nach Deutschland bringen sollten.

Zu Kriegsbeginn zählte Metz 48000 Einwohner, eine Zahl, die sich bis zur Einschließung noch um 28000 Flüchtlinge erhöhte ${ }^{4}$. An Soldaten, zumindest jene der Festungsgarnison, die damals mit 9000 Mann sowohl im französischen als auch im deutschen Vergleich ungewöhnlich hoch lag5, war man gewöhnt. Das Kommen und Gehen von Truppen sowie deren Kampieren in

1 Zit. nach Braudel, Frankreich, Bd. III, S. 344.

2 Ibid S. 346.

31850 war die älteste Eisenbahnlinie Lothringens zwischen Metz - Novéant - Pagny eröffnet worden, 1852 die Strecke Paris - Metz - Saarbrücken. Vgl. LiNCK, Verkehrswesen, S. 177. PARISSE, Lothringen, S. 514.

${ }^{4}$ Histoire de la population, S. 215. Die Angabe der Flüchtlinge bei BazAINE, L'Armée, S. 229. PAulus spricht von 75000 Einwohnern inklusive Flüchtlingen. Vgl. DERs., Cernierung, S. 57.

${ }^{5}$ Die französischen Grenzfestungen waren insgesamt stärker besetzt als die deutschen, doch lagen die Zahlen hier wie dort nirgends höher als 3000 . Vgl. BRAUDEL, Frankreich, Teil I. SICKEN, Landstreitkräfte, S. 116. 
Stadt und Umgebung gehörte lange vor der Einrichtung einer ständigen Besatzung zum Alltag der Bewohner. Besonders gefürchtet war die Zeit des Winterquartiers, wenn Massen an Soldaten und Pferden Unterkunft suchten $^{6}$. Dann wurden auch privilegierte Metzer Bürger gezwungen, Türen und Speicher zu öffnen.

Die Stadt ist älter als die Festung und weist eine Siedlungskonsistenz seit der Antike auf. In römischer Zeit befestigt und zeitweise vermutlich bereits von einer Garnisonstruppe belegt ${ }^{7}$, wird Metz im 4. Jahrhundert Bistum. 451 von den Hunnen eingeäschert - Attila soll nur ein einziges Gotteshaus verschont haben -, ersteht der Ort unter den Merowingern rasch zu neuer Größe. Unter diesen 511 Hauptstadt und Königssitz des Teilreiches Austrasien, wird er zur Wiege des karolingischen Geschlechts. Nach dem Vertrag von Mersen (870) kommt Metz zum ostfränkischen und späteren deutschen Reich und entwickelt sich zu einem der Dreh- und Angelpunkte innerhalb der so oft von politischen Wechselfällen betroffenen Gegend zwischen Rhein und Kanalküste. Der Name Lothringen, der ursprünglich für den gesamten Landstrich gilt, geht auf die kurze Zwischenreichszeit unter Lothar I. zurück. Seit den fränkischen Eroberungen immer wieder Schauplatz politisch folgenschwerer Kriege ${ }^{8}$, bilden sich hier zugleich bedeutende herrschaftskulturelle und wirtschaftliche Zentren heraus. Nicht zufällig ist gerade dieser von den Alpen bis Flandern reichende Landstrich heute das Herzstück eines friedlichen Westeuropa.

Am Beginn des 13. Jahrhunderts ist Metz freie Reichsstadt und gilt als chambre de l'empire. Zugleich beginnt man mit dem Bau der französich-gotischen Kathedrale9. Auch französische Literatur bleibt weiter vorherrschend - altfranzösische Epik und die Chansons der Geste des Lorraines, die zum Saitenspiel der von Burg zu Burg ziehenden Jongleurs erklangen, werden hier, in der admirable cité der französischen Dichter, niedergeschrieben. Der Zyklus der Heldenlieder vom Rolands- über das Garinlied bis zum Hervis de Mes hält die Erinnerung an Austrasien wach, das vom Rhein bis an die Marne reichte und dessen Hauptstadt Metz war ${ }^{10}$. Vom Bistum, das über mannigfaltigeren Besitz als Trier verfügt, strahlt der katholische Geist auf ganz Lothringen aus. Doch tritt das Kulturelle bereits in den Hintergrund: Metz wird zur Handels- und Geldmetropole zwischen Ost und West, zählt

6 Braudel, Frankreich, Teil I, S. 347.

7 LANGSDORF, Militärstadt, S. $531 \mathrm{f}$.

8 Hierher gehört der für das moderne, nationalstaatliche Frankreich so wichtige Sonntag von Bouvines im Sommer 1214 und die damalige Flucht des deutschen Kaisers Otto IV. vor dem französischen König Philipps II. August; sodann wichtig die Schlacht bei Azincourt 1415 und die übermächtigen englischen Bogenschützen, der Tod Karls des Kühnen in der Schlacht bei Nancy und das Ende des Burgundischen Zwischenreiches oder die Weltminute von Waterloo, die Blücher kommen und Napoleon verzweifelt auf seinen General Grouchy warten sah.

9 Schmitz, Metzer Kathedrale, S. 416-418.

10 STÄhlin, Geschichte Elsaß-Lothringens, S. 21. PARISSE, Lothringen, S. 184-186. 
zeitweise an die 80000 Einwohner und gehört zu den reichsten Städten der Zeit mit einem blühenden Handwerk. Bald ist der gesamte lothringische Adel vom Herzog bis hinab zum kleinen Ritter verschuldet, was Gründe für mancherlei Fehde liefert, in deren Folge sich die Stadt nicht selten zu immensen Geldzahlungen gezwungen sieht, um Unheil von ihren Mauern abzuwenden; so 1444, als sie durch Karl VII. und René II. von Anjou längere Zeit belagert wird ${ }^{11}$. Überhaupt haben Stadt und Hausbesitzer, und hier vor allem die patrizische Oberschicht, auch in Friedenszeiten immer wieder durch Steuern und Geldmittel militärischen Zwecken zu dienen - sei es zur Verköstigung der Soldaten oder nur zu Baumaßnahmen, die seit dem 16. Jahrhundert meist auf eine militärisch motivierte Veränderung des Stadtbildes zielen ${ }^{12}$. Auch für Metz spielen wirtschaftliche Expansionsmöglichkeiten eine wichtige Rolle, doch ist es auf Dauer das militärstrategische Element, das die Bedeutung des Ortes ausmacht ${ }^{13}$.

Reichsunmittelbar bleibt der Platz bis ins Jahr 1552, das einen tiefen Einschnitt für Stadt und Grenzland bedeutet. Dabei darf man sich die Grenze noch nicht als durchgehende Linie denken, sondern „als breiten in der Regel schlecht gezogenen Strich" mit Ex- und Enklaven und Territorien, die sich in- und übereinander schieben ${ }^{14}$. Dennoch färbt damals so etwas wie ein erstes nationales Moment die dynastischen Ränkespiele wie das städtisch-bürgerliche Leben neu ein und zieht entsprechende Geschichtsbetrachtungen nach sich ${ }^{15}$. Heinrichs II. Voyage d'Allemagne bringt die Bischofsstädte Verdun, Metz und Toul sowie dazugehörige Teile Lothringens an Frankreich, nachdem die evangelischen Reichsfürsten, allen voran Moritz von Sachsen, dem französischen König die Schutzherrschaft angetragen und ihre tatkräftige Unterstützung zugesagt haben ${ }^{16}$. Als Karl V. seine Söldner zur Rückeroberung ins Feld schickt, entbrennt ein erbitterter Kampf um die Reichsstadt. Es ist nicht die erste, aber die historisch bedeutsamste Belagerung jener Frühzeit, die Metz zu bestehen hat. Seither umworbenes Streitobjekt innerhalb eines unruhigen Grenzlandes, gehört zum europäischen Charakterzug der Stadt mithin ihre Lage im Spannungsfeld zunächst dynastischer und später nationaler Konflikte. Damals berennen die Spanier noch vergeblich die Mauern, auf denen sich die Franzosen unter Franz von Guise mit Erfolg behaupten. Von Hunger und Frost zermürbt, müssen die Belagerer zu Jahres-

11 Im Streit mit den Königen von Sizilien bzw. Frankreich blieb Metz standhaft, mußte aber hohe Tribute zahlen, um sich seine Freiheit zu erkaufen. CosTER, Stadt und Festung, S. 34-36.

12 KeUNe, Wandlungen des Stadtbildes, S. $368 \mathrm{f}$.

13 Henri Pirenne hat zuerst die wirtschaftliche Bedeutung mittelalterlicher Städte betont, dabei lediglich Residenz- und Hauptstädten einen Sonderstatus eingeräumt, militärische und militärstrategisch wichtige Orte hingegen vernachlässigt. Vgl. PIRENNE, Sozial- und Wirtschaftsgeschichte, S. $163 \mathrm{f}$.

14 BraUdel, Frankreich, Teil 1, S. $338 \mathrm{f}$.

15 WestPHAL, Metz, Teil I, Vorwort.

16 Dazu ausführlich ZeLLER, La réunion de Metz, Bd. I, S. 285-392. 
BELAGERUNG vom METZ DUNEN KAISER KAAL V, 1552, nach dem Cruquis von Fénelon Salignac, welches die l̈berschrift frihrt:

Le plant de la ville de Mets, felon fa vraye proportion.

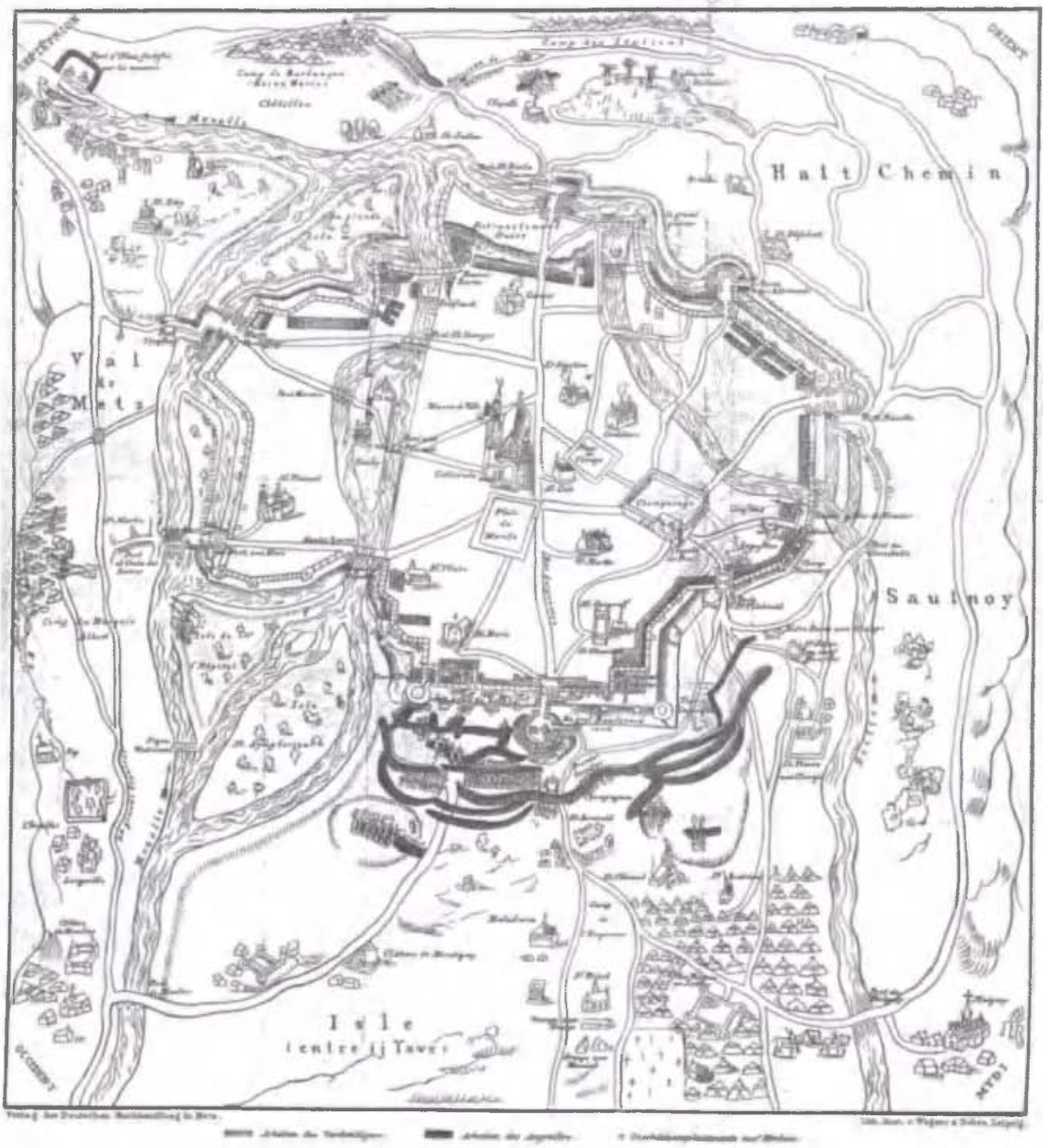

Abb. 1: Belagerung 1552 zu Metz. Quelle: WestPhal, Geschichte der Stadt Metz. 
ende aufgeben. Grund dafür ist sinnfälligerweise eine gute Vorbereitung der Verteidiger in allem Wesentlichen, vor allem eine ausreichende Verproviantierung des Platzes durch gewaltsame Requirierungen ${ }^{17}$ und die systematische Verwüstung umliegender Vororte, Abteien, Kirchen, Kapellen, Schlösser und Höfe wie einzelner Stadtteile selbst. Militärische Zwänge schneiden hier erstmals seit römischer Zeit wieder scharf in den zivilen Bereich ein ${ }^{18}$. Vom Ereignis angestoßen, wird Metz jetzt mit dem Bau einer Citadelle (1556-62) fortifikatorisch verstärkt, wobei das Bollwerk die Stadt mehr und mehr einzuschnüren beginnt, Wohnraum, Kirchen und Kirchtürme „niedergelegt", also vernichtet werden. Während die umliegenden Dörfer immer von Handstreichen und Plünderungen bedroht bleiben, sichert sich die Festung so gegen überraschende Angriffe ab. Militär und Zivilisten kommen in der Stadt miteinander aus, aber draußen toben auch weiterhin fast ununterbrochen die Kämpfe zwischen durchziehenden Soldaten und ansässigen Bau$\mathrm{ern}^{19}$. Im Dreißigjährigen Krieg Durchgangslager für protestantische Söldner, ist die Stadt mit 12-15000 Hugenotten ein Refugium für französische Glaubensflüchtlinge und Protestanten. Deren Abwanderung, die bis zum Ende des 17. Jahrhunderts erzwungen ist, bedeutet auch eine Abschwächung des Deutschtums in der Region ${ }^{20}$. Wie ganz Lothringen, so erfährt auch Metz nach dem Frieden von Münster und vielerlei Wechselfällen eine scharfe, vor allem durch die Jesuiten vollzogene Rekatholisierung. Es gehört nun auch offiziell zu Frankreich, dessen Ansprüche damit aber keineswegs befriedigt sind $^{21}$. Um die Festungsstadt flaut der Krieg jetzt für längere Zeit ab. Seither nicht mehr ernsthaft angefochten, bekommt man den Feind selbst im spanischen Erbfolgekrieg nur noch von weitem zu Gesicht und entwickelt sich zur Garnisonsstadt im modernen Sinne. Neben die Bürgerwehr, der traditionell Männer aus Stadt und Land sowie eine Jugendwehr angehören, tritt eine aus Berufssoldaten zusammengesetzte ständige Garnison ${ }^{22}$. Berühmte Militärs wie die Generale Fabert, Custine oder Kellermann gehen aus ihr hervor. Vauban, Cormontaigne und deren Nachfolger verstärken den Waffenplatz seit Ende des 17. Jahrhunderts - man baut nach italienischem Vorbild im bastionierten Grundriß mit stumpf vorspringenden Winkeln - vornehmlich als Ausgangspunkt für Offensivaktionen über den Rhein hinaus nach Osten, vor allem nach Süddeutschland. Andererseits soll Metz, wie Turenne bemerkte,

im Unglück und nach verlorenen Schlachten als Zufluchtsort dienen, um ein Heer aufzunehmen, den Nachbarländern zu helfen und die Verkehrswege im Hinterland zu

17 WestPhal, Metz, Teil II, S. $17 \mathrm{f}$.

18 KEUNE, Wandlungen des Stadtbildes, S. 358.

19 BRAudel, Frankreich, Teil I, S. 348 f.

20 STÄHLIN, Geschichte Elsaß-Lothringens, S. 146. Es blieben lediglich 1700 Einwohner zurück, die zum Katholizismus konvertiert waren. THIRIOT, Metz als französische Provinzhauptstadt, S. 350.

21 Vgl. SCHMIDT, Der Dreißigjährige Krieg, S. 77.

22 LANGSDORF, Militärstadt, S. 537. 
schützen. Unter Umständen könnte allein diese Festung die vereinigten Kräfte des Reiches aufhalten ${ }^{23}$.

Im Herbst 1870 wurde hierzu die Probe aufs Exempel gemacht. Nach und nach werden unter Vauban und seinen Nachfolgern freie Plätze mit Kasernen, Lazaretten und Magazinen gefüllt ${ }^{24}$. Augenscheinlich ist der Wandel vom religiösen und politischen Zentrum zum Militärstandort. Metz wird von der stetig wachsenden Garnison vereinnahmt und kommt schrittweise unter deren Aufsicht ${ }^{25}$. Die modernisierte Festung beginnt die Stadterweiterung zu bremsen und ist auch sonst ein eher urbanisierungshemmender Faktor, der die Lage noch bis zu Beginn des 20. Jahrhunderts bestimmt ${ }^{26}$.

In der Revolution ein Zentrum der Jakobiner, gerät der Platz auch während der napoleonischen Kriege nicht ernsthaft in Bedrängnis. Selbst 1814/15 begnügen sich die Verbündeten damit, Metz lediglich für kurze Zeit blockieren und beobachten zu lassen, nicht ohne es schließlich doch zu zwingen, die weiße Fahne zu hissen ${ }^{27}$. Inzwischen sind in der Metzer Ecole de l'artillerie et du genie die vormaligen Militärschulen von Mézières und Chalons vereint, was den Garnisons- und Festungsstandort weiter aufwertet. Die Verwaltungs- und Unterrichtsgebäude der neuen Schule werden auf den Fundamenten der in der Revolution niedergerissenen Kirche der Großkarmeliter errichtet ${ }^{28}$. Letzte fortifikatorische Verbesserungen erfährt die Festung nach dem Paukenschlag von Sadowa ${ }^{29}$, der die Franzosen in erhöhte Alarmbereitschaft versetzt. Durch den Bau mehrerer neuer Forts wird sie nun zu einem verschanzten Lager ausgebaut, das im August 1870 zwar noch an vielen Stellen unfertig ist, aber modernen Standards genügt ${ }^{30}$. Zuletzt verharrt sie in der „Hab-Acht-Atmosphäre“31 eines über lange Jahre unbesiegten, selbstbewußten Ortes, dessen Verteidigungsfähigkeit durch die große Belagerungs-

23 Braudel, Frankreich, Teil I, S. 344 f. Vgl. auch Friedrich EngELs, Das Schicksal von Metz, in: MEW, Bd. 17, S. 137-140.

24 Ibid S. 360-364.

25 Vgl. Tippach, Garnison, S. $243 \mathrm{f}$.

26 Das oft befruchtende und vor allem infrastrukturell produktive militär-zivile Verhältnis einfacher Garnisonsstädte scheint es für Metz wegen seines spezifischen Festungscharakters und seiner brisanten Lage über weite Strecken der neuzeitlichen Entwicklung offenbar nicht gegeben zu haben. Dazu KEuNE, Veränderungen des Stadtbildes. Folz, Metz als deutsche Bezirkshauptstadt, S. 375 f. WITTENBROCK, Stadterweiterung, S. If. Ähnliche Erkenntnisse für Koblenz bei TIPPACH, Garnison, S. $254 \mathrm{f}$.

27 Coster, Stadt und Festung, S. 135. PARISSE, Lothringen, S. $389 \mathrm{f}$.

28 LANGSDORF, Militärstadt, S. 540 f. KEUNE, Wandlungen des Stadtbildes, S. $369 \mathrm{f}$.

29 In Frankreich namensgebend für die Schlacht bei Königgrätz (3. Juli 1866), wo Preußen über die verbündeten österreichisch-sächsischen Truppen triumphierte. „Rache für Sadowa" hieß es fortan in Paris, dessen Kompensationswünsche nach der Gründung des Norddeutschen Bundes von Bismarck zurückgewiesen worden waren.

30 FIRCKS, Vertheidigung, S. 14-17. Paulus, Cernirung, S. 50-53. Zum Bau der Forts in Wort und Bild: DENIS, Garnison, S. 113-116.

31 Braudel, Frankreich, Teil I, S. 343. 


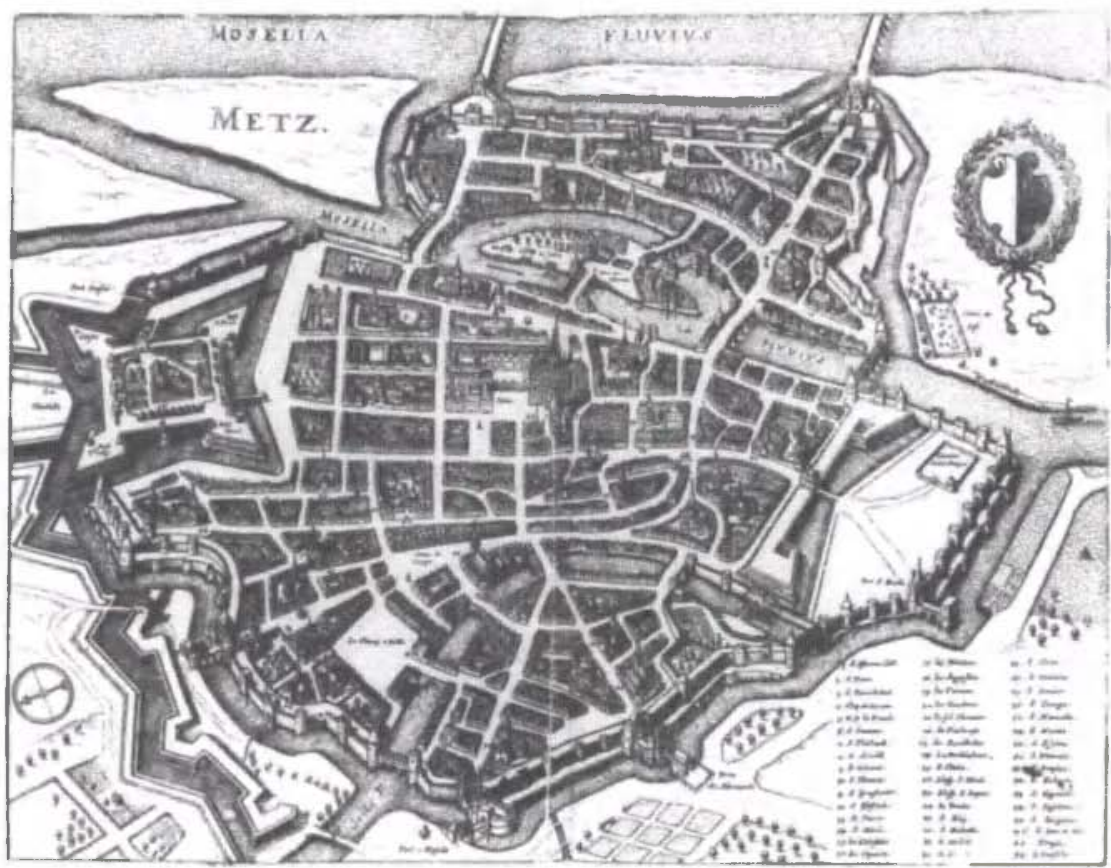

Abb. 2: Festungsstadt um die Mitte des 17. Jahrhunderts. Quelle: Topographia Palatinatus Rheni et Vicinarum Regionum. An Tag gegeben und verlegt durch Mattheum Merian 1645.

übung von 1844 nochmals spektakulär zur Schau gestellt worden war ${ }^{32}$ - anwesend damals auch ein Prinz von Sachsen-Weimar und 19 preußische Offiziere $^{33}$. Als etwa zur selben Zeit ein großes Manöver auf dem Schlachtfeld von 1806 bei Jena stattfand, soll der preußische König zu einem französischen Beobachter bemerkt haben: "Sehen Sie, heute haben wir gewonnen." Darauf der Gast: „Mein Herr, damals wurde scharf geschossen. “ Für Metz mußte es sich im August 1870 erweisen, ob die in Friedenszeiten gemachten Erfahrungen auch für den Ernstfall ihre Gültigkeit behalten sollten.

Zu Kriegsbeginn galt Metz als stärkstes Glied innerhalb des französischen Festungsgürtels zwischen Dünkirchen und Belfort, entlang der belgisch-luxemburgisch-deutschen und der Schweizer Grenze. Doch war die Südflanke, deren Eingangstor und zugleich neuralgischen Punkt die Stadt bildete, schwächer als der dichter gestaffelte Festungsbezirk weiter nördlich ${ }^{34}$. Auch lag sie genau in jener Breite des lebendigsten und umstrittensten Stücks fran-

32 Coster, Stadt und Festung. Langsdorf, Militärstadt, S. 539.

33 DenIs, Garnison, S. 175.

34 BRaudel, Frankreich, Teil I, S. 338. 


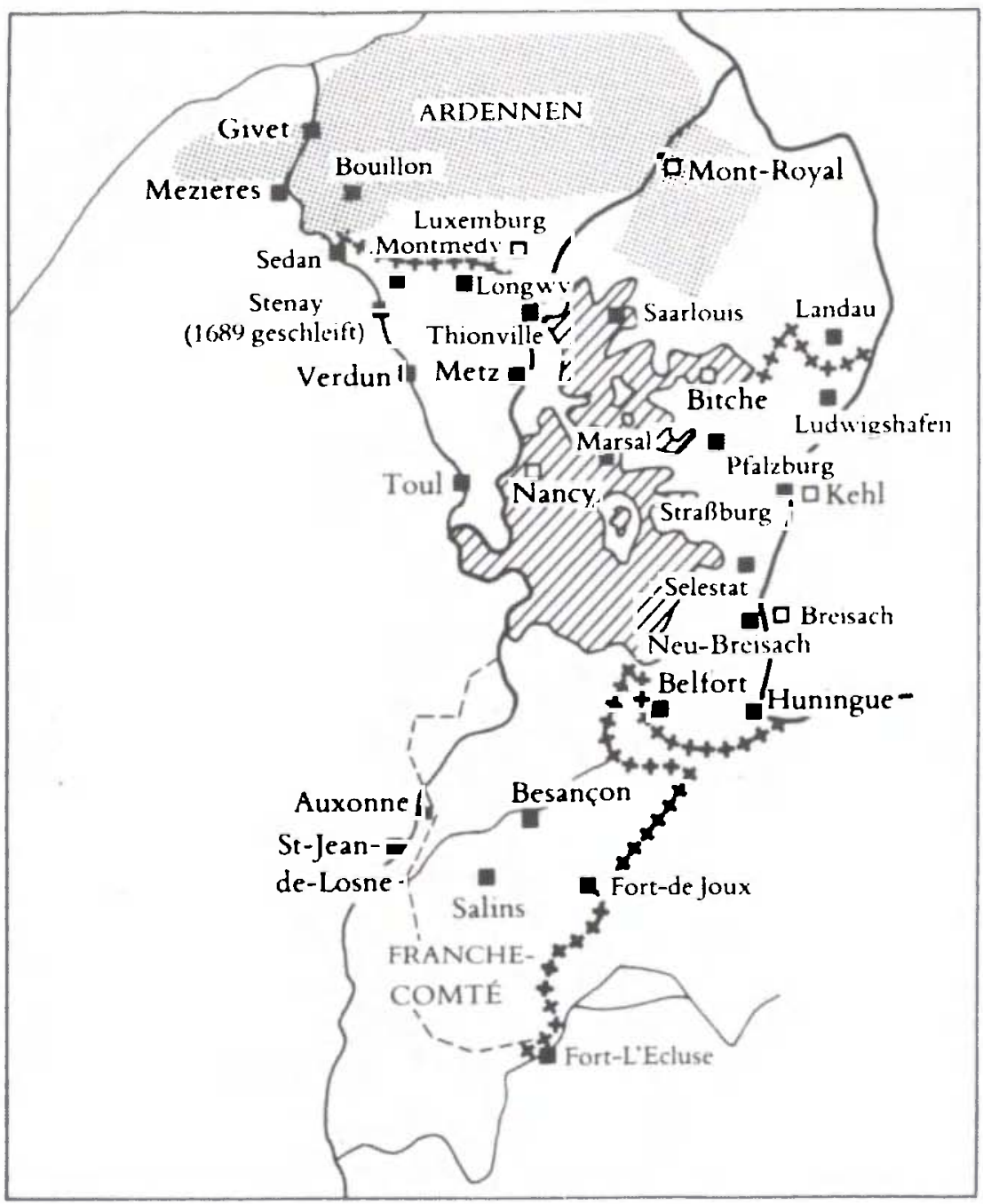

Die Festungen der Ostgrenze

Die verlorenen (weißes Quadrat) und die verteidigten Festungen (schwarzes Quadrat) an der besonders "sensiblen" Grenze der Ardennen, Lothringens, des Elsaß und der Franche-Comte während der endlosen Kriege des 17. und 18. Jahrhunderts.

$\square$ Stellung verloren

- Stellung verteidigt

Abb. 3: Die Festungen der Ostgrenze. Quelle: Pierre Rocolle, 2000 ans de fortification française, Paris 1973. 
zösischer Ostgrenze, das von Feinden öfter durchbrochen worden war, um ins Landesinnere vorzustoßen ${ }^{35}$. Koblenz bildete auf deutscher Seite das Pendant dazu. Zwar erst nach 1815 durch die Preußen zur starken Festung ausgebaut und auch erheblich schwächer besetzt, kam der Stadt infrastrukturell und militärstrategisch eine ähnlich herausragende Stellung zu wie Metz auf der anderen Seite: als Rhein-Moselfestung und urdeutscher Platz, nach dem der französische Arm öfter gegriffen hatte, sowie als Eisenbahnknotenpunkt und Wirtschaftsstandort in Grenznähe ${ }^{36}$.

35 Ibid.

36 TIPPACH, Garnison, S. 243 f. Ein Vergleich wäre reizvoll. 
